\title{
Tuberculosis Unexpectedly Presented Along Gastrointestinal Tract in Surgical Ward
}

\author{
Noori H. Jasim ${ }^{1, ~ *, ~ H a s h i m ~ S . ~ K h a y a t ~}{ }^{2}$ \\ ${ }^{1}$ General and GI Surgery, Department of Surgery, Faculty of Medicine, University of Basra, Basra, Iraq \\ ${ }^{2}$ Basra General Hospital, Basra, Iraq
}

Email address:

noorihanon@yahoo.com (N. H. Jasim)

${ }^{*}$ Corresponding author

\section{To cite this article:}

Noori H. Jasim, Hashim S. Khayat. Tuberculosis Unexpectedly Presented Along Gastrointestinal Tract in Surgical Ward. Journal of Surgery. Vol. 5, No. 1, 2017, pp. 1-7. doi: 10.11648/j.js.20170501.11

Received: February 3, 2017; Accepted: February 21, 2017; Published: March 15, 2017

\begin{abstract}
Little is known in our country particularly in Basra about tuberculous lesions affecting the gastrointestinal tract specifically solid and hallow organs such as liver, pancreas, stomach or intestine. Hence, this study aimed to report, describe and study these cases. The current study is retrospectively studied 12 patients who got tuberculous lesions along the gastrointestinal tract over the last 2 decades in Basra General Hospital (2 of them were gastric, 3 hepatic, 3 pancreatic, 3 small intestinal; one of them affecting duodenojejunal flexure, and lastly 1 perianal sinus ulcerating the nearby skin). All those patients underwent the required surgical operations. In all those patients, TB was diagnosed postoperatively by histopathology and all of them received anti-tuberculosis triple therapy for 6 months including INH, Rifampicin and Ethambutol. A long term scheduled follow up was done showing that all of them were improved except one elderly patient with TB pancreatitis died at early postoperative period. None of these cases effected with pulmonary tuberculosis previously or concomitantly. One case of hepatic tuberculosis had cervical and intra-abdominal lymphadenopathy. It is of interest to mention that extrapulmonary TB in solid and hallow organs of gastrointestinal tract should be considered when evaluating patients with gastrointestinal presentations and lesions such as ulcer, mass, abscess or perianal fistula in endemic areas and the potential for affecting these organs (along gastrointestinal tract) should be kept in mind and accounted for.
\end{abstract}

Keywords: Tuberculosis, Gastrointestinal Tract, Surgical Ward

\section{Introduction}

Tuberculosis (TB) is a common disease in the world affecting one third of whole population [1] particularly in developing countries. [2] It is caused by Mycobacterium tuberculosis. [3] It is known that the lung is commonest site affected by TB. [4] However, TB may affect different parts of body in presence or absence of pulmonary tuberculosis. [4] Most tuberculous infections are symptomless and called latent tuberculosis. Approximately 10\% of latent tuberculosis progresses to active tuberculosis. [3] Extrapulmonary TB occurs most notably in pleura, central nervous system, lymphatic system particularly in the neck, genitourinary system, bone and joints and sometimes skin resulting in tubeculous skin ulcer. [5], [6] Extrapulmonary TB may present simultaneously with pulmonary TB. [4] Extra pulmonary TB represents less than $15 \%$ of all cases of tuberculosis [7], [8] whereas the intestinal one represents less than $1 \%$ of all extrapulmonary TB cases. [9]

Gastrointestinal TB is relatively less frequent in US and western countries in comparison to certain area in Asia and Africa and become mostly restricted in US to immigrants come from endemic areas and AIDS patients. [10], [11] In addition, strict measures of milk pasteurization have approximately eliminated bovine tuberculosis in many regions in the world. [10] In our locality, the primary responders to the cases of gastrointestinal manifestations are the doctors of primary health centers, private clinics, outpatient clinics, and the emergency departments; these doctors are of variable specialty and experience. In addition, in many patients, there was a delay in seeking medical attention. All these factors may cause a delay in the treatment of gastrointestinal TB cases leading to complications such as 
partial obstruction or perforation of intestine, mass in liver or pancreas, perianal fistula and ulceration. Furthermore, our country is endemic with pulmonary TB, in spite of that, little is known and reported about gastrointestinal tuberculosis involving solid and hallow organs particularly in Basra. Hence, this research work interestingly aimed to report, describe and examine different cases of TB of different organs of gastrointestinal tract.

\section{Patients and Methods}

It is a retrospective study includes 12 patients presented with gastrointestinal symptoms due to lesions caused by tuberculosis like gastric ulcer, intestinal mass and stricture, pancreatic and hepatic mass or abscess and perianal sinus and ulceration, and who had underwent the required surgery over the last two decades in Basra General Hospital. Histopathological exam proved the diagnosis of tuberculosis in the excised tissue lesions or biopsies in all patients. Chest $\mathrm{X}$-ray films of all patient were normal and there was no evidence of pulmonary TB. One patient had a history of cervical and intra-abdominal tuberculous lymphadenitis and showed resistance to anti-tuberculosis treatment. Then, all patients received anti-tuberculosis triple therapy, after removal of stiches, in Basra Tuberculosis Centre for 6-9 months. Patients were seen immediately after finishing their anti-tuberculosis treatment and then after one year. In both visits, patients were well except one elderly patient with pancreatic TB died in early postoperative period.

The followings are short presentation of these cases:

Case $1 \& 2$ (Gastric cases)

Two male patients (40 years and 36 years old) presented with epigastric pain for few weeks not responding to medical treatment in terms of proton pump inhibitor followed by repeated vomiting for the last few days before admission to the hospital. Upper gastrointestinal endoscopy was done for both of them finding dirty ulcerative lesions in both. Endoscopic biopsies were taken for them for histopathology and the results were inconclusive in both and surgery was decided. Preoperative preparation was done including all required blood investigations, ECG and chest X-ray. Partial gastrectomy with gastro-duodenostomy was done for both. Resected segments were sent for histopathology revealing the diagnosis of 'tuberculous gastritis'. Both patients stayed few days in hospital and discharged home well.

Case 3, 4 \& 5 (Hepatic cases)

Patients (case 3 and 4) were both of them male (55years and 62years old). Both presented with right upper abdominal pain, fever, malaise and anorexia for few days. They were unwell. Ultrasonic scan showed space occupying liver lesions with necrotic core in right lobe of liver. Preoperative preparation was done including all required blood investigations, ECG and chest X-ray. Surgery was done using right subcostal incision finding a liver lesion in right lobe of liver. Deroofing of liver lesion was performed and necrotic tissue and debris was removed and sent for histopathology. This test proved the diagnosis of 'tuberculous liver abscess'.
Both patients stayed few days in hospital and discharged home well.

In case 5 , the patient was girl of 6 years old. She had a history of cervical tuberculous lymphadenitis followed by intrabdominal tuberculous lymphadenitis proved by histopatholgical exams showing multiple epithiliod granulomas with extensive caseous necrosis and Langhan's giant cells (figure 1A). This patient hasnot responded to antituberculosis triple therapy for few months and ultimately presented to our hospital while the patient still complaining of anorexia, weight loss, night sweeting and right upper abdominal pain. Abdominal ultrasound and then CT scans were done and showed an ill-defined irregular hypointense mass lesion with peripheral calcification in left lobe of liver suggesting TB granuloma (tuberculoma) (figure 1B). Chest $\mathrm{X}$-ray and CT scan showed no evidence of pulmonary tuberculosis (figure 1C). Preoperative preparation was done including all required blood investigations, ECG and chest X-ray. Surgery was done using right subcostal incision finding a big liver lesion in left lobe of liver in addition to few nodules spread along the surface of both lobes of liver (figure 1D). This lesion was deroofed and evacuated (figure $1 \mathrm{E})$ and pus was aspirated and sent for bacteriological exam for acid fast bacilli (figure 1F). Necrotic material was removed and sent for histopathological exam. Both test proved the diagnosis of tuberculous lesion in liver.

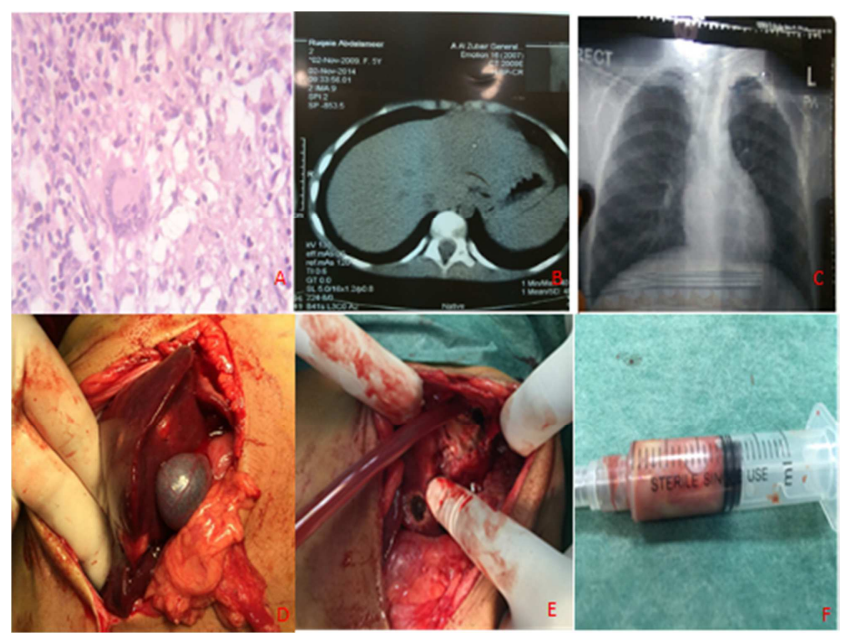

Figure 1. Shows a 6 years old girl with tuberculous hepatitis. (A): Patient had a history of cervical and intra-abdominal Tuberculous lymphadenitis proved by histopathology (400X). (B): abdominal CT scan shows an illdefined irregular hypointense mass lesion with peripheral calcification in left lobe of liver suggesting TB granuloma (tuberculoma). (C): The chest $X$ ray is normal for pulmonary $T B$. (D): Surgery using right subcostal incision shows a big liver lesion in left lobe of liver in addition to few nodules spread along the surface of both lobes of liver. (E): The lesion was deroofed and evacuated. $(F)$ : Shows the color of pus aspirated from the lesion.

\section{Case 6, $7 \& 8$ (Pancreatic cases)}

Patients were one male patient of 26 years old and the other two patients were female ( 22 years and 70 years). All patients presented with low grade fever, weight loss and upper abdominal pain. Ultrasound scan showed a pancreatic mass at the body of pancreas with suspicion of malignant 
tumor. preoperative preparation was done as usual and there was no evidence of pulmonary tuberculosis in their chest XRays. Laparotomy was done showing pancreatic body mass. These masses were palpated in order to assess its respectability finding that these masses are soft friable with yellow caseation appearance in its central part. The debris was excised and cleansing was done. Histopathological exam showed 'tubercolous pancreatitis'. All patients stayed few days in hospital and discharged home well except the elderly lady (lady of 70 years) died at third post-operative day.

Case 9, 10 \& 11 (Small Intestinal cases)

One patient (female of 43years) presented as acute abdominal condition with generalized abdominal pain and generalized tenderness with rebound tenderness beside rigidity all over the abdomen with tachycardia and hypotension. Ultrasound scan showed a moderate amount of free fluid in abdominal and pelvic cavity. Explorative laparotomy was done showing a perforation in terminal ileum with free fluid. Biopsy was taken from perforation site for histopathology. Suction of free fluid and debridement of site of perforation with primary suturing was performed alongside proper washing of abdominal and pelvic cavity. This patient stayed few days in hospital and discharged home well. Histopathological exam proved the diagnosis of small intestinal tuberculosis.

Other patient (female of 41 years old). She presented as case of partial intestinal obstruction including generalized cramps in the abdomen with loud borborygmi, few attacks of vomiting associated with abdominal tenderness particularly at right lower abdomen. Ultrasound scan revealed presence of thickened bowel loops. Explorative laparotomy was done finding a small intestinal mass at a terminal ileum consisting of an ileal stricture surrounded with adherent omentum and affected mesentry with matted lymph nodes beside inflamed adjacent tissue. The mass was properly dissected and the affected segment of small intestine was resected and end to end anastomosis was done. Histopathological exam showed a tuberculous stricture of small intestine. The patient was discharged home well after few days staying in the hospital.

The third patient in this group (male of 52 years). He presented as a vague upper abdominal pain and bouts of vomiting. Barium study showed area of narrowing in small intestine (duodeno-jejunal junction). Patient was preoperatively prepared as usual and chest $\mathrm{X}$ ray was normal. Explorative laparotomy was done finding a small intestinal mass in duodeno-jejunal junction consisting of small segment of small intestine with adherent omentum. The affected segment was resected and then, one end of intestine was anastomosed to the other end. Histopathological exam showed a tuberculous small intestinal stricture. The patient was discharged home well after few days staying in the hospital.

Case 12 (perianal)

This is a 50 years old male patient presented with dirty perianal ulceration. There was no history of pulmonary or other organ TB and no history of previous perianal abscess or infection. His general examination showed that the patient was well. The perianal inspection revealed large unilateral dirty ulcer. The digital per rectal and proctoscopic exam showed a perianal sinus with nodular wall. MRI showed presence of a 2.5 perianal sinus tract with external extent close to the perianal skin with ill-defined enhancing inflammatory changes were noted surrounding the perianal tract (figure 2A). After proper patient preparation, debridement of the perianal lesion was done and then the area was cured. Histopathological examination of biopsies showed tuberculous granuloma with presence of Langhan's giant cells (figure 2B). The patient discharged home well in the second post-operative day.

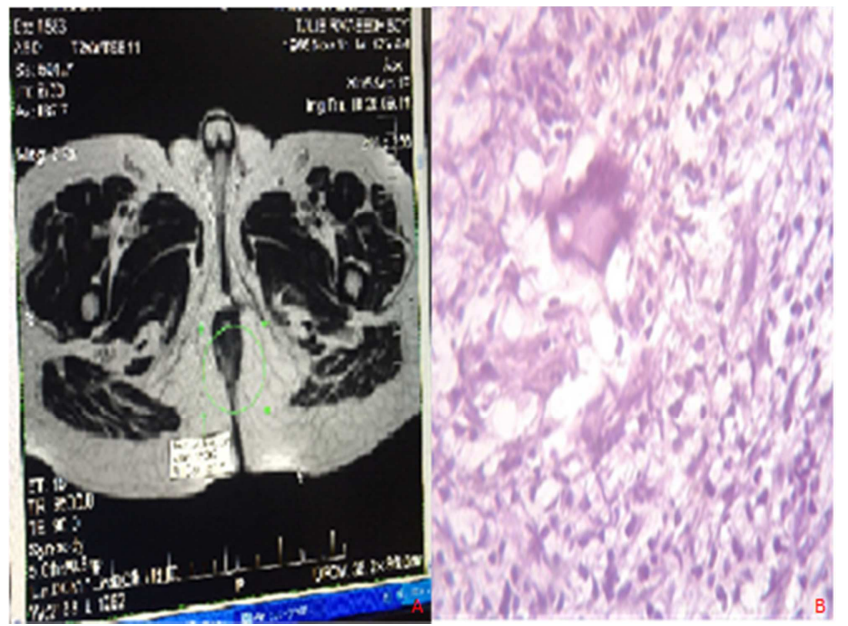

Figure 2. Shows (A): MRI of patient reveals presence of a 2.5 perianal sinus tract with external extent close to the perianal skin with ill-defined enhancing inflammatory changes were noted surrounding the perianal tract. (B): Histopathological examination of biopsies showed tuberculous granuloma with presence of Langhan's giant cells (400X).

\section{Results}

Over the period of study (about 2 decades), 12 patients were included in this study. All of them had tubercolous lesions along the gastrointestinal tract. They were 7 males and 5 females with male to female ratio (1.4). Their age ranged from 6 years to 70 years with mean age 37.85 years.

In two of them, TB affected the stomach 'tuberculous gastritis" and presented as gastric ulcer with epigastric pain which was diagnosed by upper gastrointestinal endoscopy. In addition, in the other three patients, the liver was affected with TB leading to mass and abscess lesions and patients presented with right upper abdominal pain, fever, malaise and anorexia and diagnosed by ultrasound and CT scans as shown in table (1). Furthermore, in other 3 patients pancreas was affected with TB and presented as pancreatic mass with non-specific presentations such as low grade fever, weight loss and upper abdominal pain and the pancreatic mass was detected by ultrasound scan. Moreover, in other three cases, small intestine was affected with TB and the presentations were in two of them as small intestinal mass with signs and symptoms of partial small intestinal obstruction in terms of abdominal pain and repeated vomiting whereas in the other one as acute abdomen with generalized abdominal pain, 
tenderness, rigidity and tachycardia. Lastly, in one patient, perianal region was affected with $\mathrm{TB}$ and patient presented with perianal sinus and dirty ulceration.

In all patients, chest X-Ray was normal and there was no evidence of pulmonary TB. However, one patient had a disseminated tuberculous lymphadenitis involving both cervical and intra-abdominal lymph nodes, and she was refractory to anti TB therapy before her liver being affected with TB resulting in a liver tuberculous abscess.

Tuberculosis, in all these cases, were diagnosed by histopathological exam and in one case by bacteriological exam for detection of acid fast bascilli beside histopathological exam as shown in table (1). Then, all patients in our series (Except one with pancreatic TB who died at early post-operative period) received anti-TB therapy including INH, rifampicin and ethambutol for 6-9 weeks in Basra Tuberculosis Centre. All patients who received anti-TB therapy were improved except one who is still in the beginning of her second course of anti-TB therapy after a resistance to the first course.

Table 1. Summary of the cases.

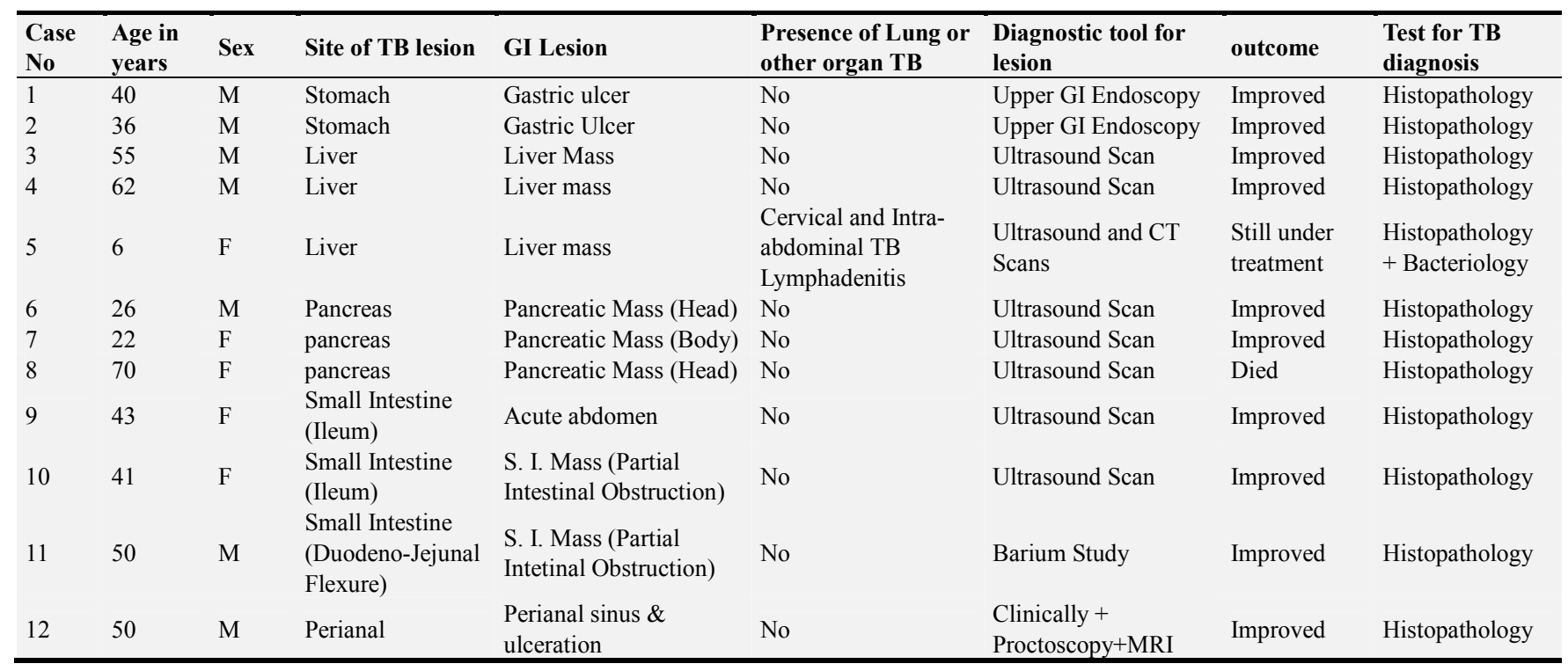

\section{Discussion}

In this study, there were 12 patients involved with gastrointestinal tuberculosis. There were 7 males and 5 females and male to female ratio is (1.4). The range of age was 6-70 years with mean age 37.85 years. A small sample size in current study might be considered as a limitation to get proper epidemiological data. In other literatures, It is reported that the epidemiology of gastrointestinal TB shows wide variation worldwide possibly attributed to several factors such as age, sex, socioeconomic factors, immunological condition, and genotype of Mycobacterium tuberculosis. [12] For example: in Pakistan, Turkey, and West Africa, young adult women are commonly affected, while in China, Singapore, India, and the United Kingdom, there is same or larger numbers of male patients affected in spite of a lower disease incidence. [12], [13]

In current study, tuberculosis affected small intestine in 3 cases (two in terminal ileum and one in duodenojejunal flexure), stomach in two cases and perianal in one case. This comes in accord with other studies that reports that the terminal ileum and ileocaecal junction are the most common site in gastrointestinal tract affected by tuberculosis, perhaps due to abundance of lymphoid tissue. [13] Then to less extent duodenum, stomach, colon, jejunm, rectum are other sites affected with TB. However, tuberculous peritonitis is still the most common form of abdominal TB. [14] The anoperineal area is rarely affected with TB and represents less than $1 \%$ of all intestinal TB. [15], [16], [17], [18]

In US, population groups at particular risk for intestinal tuberculosis are immigrants, AIDS, urban poor, the Indians on reservations. [10] Marshal JB has reported that almost 15$20 \%$ of intestinal TB have active lung TB at the same time. [10] Although, Basra considered as an endemic area with TB, none of these cases in current study had pulmonary TB and their chest X-ray were normal. This can be explained by that TB in these cases might be primary TB caused by the bovine strain due to improper measures of milk pasteurization other than secondary TB caused by ingestion of infected sputum. [14] In addition, in miliary TB, haematogenous or lymphatic spread considered as another source of intestinal TB. [9], [16] However, many other reports have described that gastrointestinal TB happens concomitant with or secondary to pulmonary TB [15], [17], [18], [19] which might be manifested thereafter or might not. [10] Nevertheless, gastrointestinal TB including Intestinal and anoperineal may develop by reactivation of the latent focus and pulmonary tuberculosis may not present. [8]

The clinical features of gastrointestinal TB (before developing complication such as obstruction or perforation) are nonspecific such abdominal pain, anorexia, weight loss, fever, night sweating and diarrhea or constipation and unless a high index of suspicion is maintained, the diagnosis can be 
missed or delayed resulting in complications. [10], [11]

In our series two cases of intestinal TB presented as partial intestinal obstruction with intestinal mass detected by ultrasound scan or barium, one patient as acute abdomen due to intestinal perforation and two cases as gastric ulcer. This could be explained by that $70 \%$ of gastrointestinal TB patients have hypertrophic type which usually occurs in ileum that might be attributed to fibroblastic reaction to the tubercle bacilli leading to stricture or adhesion or both resulting in partial intestinal obstruction in $30 \%$ of gastrointestinal TB patients. Perforation with peritonitis occurs in 5\% of gastrointestinal TB patients, usually in the ileum proximal to a stricture. Whereas only $30 \%$ of patients have ulcerative type of the disease. [20], [21], [22]

In this study, tuberculosis affected anal and perianal region in 50 years old male patient. The patient presented with perianal sinus and dirty ulceration without other pulmonary or other extrapulmonary TB in this patient. Generally, the incidence of tuberculous perianal involvement is very rare [23], [24] of approximately $0.7 \%$ of tuberculosis cases [25] and its fistulizing form more than ulcerating form. [26] Both forms should be differentiated from other serious disease (malignancy and crohn's disease). [26] Several other differential diagnosis should be taken in account such as anorectal abscesses caused by mixed bacteria, amoebiasis, sarcoidosis, syphilis, lymphogranuloma venereum, and foreign body reactions. [9], [27]

Two relatively recent case report studies have reported one case (in each study) affected by tuberculous perianal ulceration in presence of pulmonary TB simultaneously [26], [28], hence, perianal TB seems expected in these two reports and it was unexpected in current study. Furthermore, Ichihashi et al. has found that 9 out of 11 patients with perianal $\mathrm{TB}$ affected with pulmonary $\mathrm{TB}$ at the same time displaying the simultaneous occurrence and association of the two. [29] Therefore, the possible main suggested mechanism for perianal TB is secondary to pulmonary, intestinal or genitourinary TB. [26] But, hematogenous spread with subsequent infection reactivation for certain latent site of TB is more rare possible mechanism [30] and this might explain the source of TB in our case.

In this study, tuberculosis affected pancreas in 3 cases out of total 12 patients. It is reported that pancreatic tuberculosis is rare and its occurrence in immunsuppressed patients. [31] In one series, it is reported that there is only 14 cases, out of 1656 autopsies of patients with tuberculosis, had pancreatic involvement which simulate pancreatic tumors. [32] In other study from India from 1999-2004, pancreatic TB diagnosed in $8.3 \%$ of 384 patients with gastrointestinal TB. [33]

It is reported that pancreatic TB usually happened in patients with military TB (pulmonary and extrapulmonary) or primary pancreatic TB in patients with AIDS (0.46\%). [31], [32], [34], [35] In contrast, in current study all 3 patients with pancreatic tuberculosis had no military TB or AIDS. In this study 2 patients were under 30 and one was 70 . This supported by other study in which more than $50 \%$ patients with pancreatic TB were young less than 30. [33], [36]
In current study, body of pancreas was affected with TB and the diagnosis was high suspicion of pancreatic malignant tumour. However, it is reported in literatures that head and uncinate process are more commonly affected by TB and It is often missed diagnosed as a pancreatic tumor [37], [38] and this is the case in our series. Most common presentation of patients in our work, was abdominal pain, fever, weight loss as in many other previous literatures. [31] The primary site of TB was not clear in our work and this is supported by one other study. [39]

In this study, tuberculosis affected liver in 3 cases out of total 12 patients. One of them had cervical and intraabdominal TB lymphadenitis but no pulmonary TB. However, there were no pulmonary or extra pulmonary TB in the rest two patients. There are two forms of hepatic involvement with TB. Firstly, localized (isolated) hepatic TB which constitutes less than $1 \%$ of all cases of this infection. [40] Secondly, hepatic TB involvement as part of disseminated miliary TB which is much more frequent than the first type. [40], [41]

Isolated liver TB presented with clinical features attributed only to the liver lesion with minimal or no extrahepatic involvement. [41] While, hepatic involvement was reported in $80-100 \%$ of the patients of disseminated tuberculosis In one autopsy series. [42] Whereas, the localized form of liver TB is much less common. [43], [44] In addition, Kok et al. mentioned that localized liver TB represents $0.3 \%$ of 1,678 new cases of TB in their series. [45]

In miliary liver $\mathrm{TB}$, It is thought that Hematogenous spreading of mycobacterium TB bacilli is the way of hepatic infection; while, in localized liver TB, the TB bacilli possibly spread to hepatic tissue from the bowel through the portal vein. [41], [44]

The clinical features in case of liver involvement with TB are usually nonspecific including fever, night sweats, malaise, anorexia, weight loss, and abdominal pain. [40], [45] In case of disseminated $\mathrm{TB}$, presence of $\mathrm{TB}$ in other organ might give hint to diagnose liver TB. In case of absence of such clue, the diagnosis of liver TB could be highly difficult and unexpected [41] as in two of our cases of liver involvement.

Localized liver TB necessitates high degree of suspicion, and the certain diagnosis depends upon histopathological and/or bacteriological studies. [45] Histopathological examination of liver biopsy is the most dependable way. [43], [44] Imaging studies, such as CT scan, MRI and Ultrasonography, are of slight importance giving non-specific findings which are difficult to differentiate TB liver lesion from secondaries, fungal abscesses and lymphomas. [40]

\section{Conclusion}

Tuberculosis of any part of gastrointestinal tract (hallow viscus, solid organ and/or peritoneum) should be kept in account while assessing cases with variable gastrointestinal symptoms or even non-specific symptoms in endemic areas or in immunecompromized patients even in absence of 
pulmonary or elsewhere extrapulmonary tuberculosis.

\section{References}

[1] "Tuberculosis Fact sheet $\mathrm{N}^{\circ} 104 "$. World Health Organization. November 2010.

[2] "The sixteenth global report on tuberculosis World Health Organization (2011).

[3] Kumar V, Abbas AK, Fausto N, Mitchell RN (2007). Robbins Basic Pathology (8th ed.). Saunders Elsevier. pp. 516-522. ISBN 978-1-4160-2973-1.

[4] Dolin, Gerald L. Mandell, John E. Bennett, Raphael (2010). Mandell, Douglas, and Bennett's principles and practice of infectious diseases (7th ed.). Philadelphia, PA: Churchill Livingstone/Elsevier. pp. Chapter 250. ISBN 978-0-44306839-3.

[5] Kabra, [edited by] Vimlesh Seth, S. K. (2006). Essentials of tuberculosis in children (3rd ed.). New Delhi: Jaypee Bros. Medical Publishers. p. 249. ISBN 978-81-8061-709-6.

[6] Manual of Surgery. Kaplan Publishing. 2008. p. 75. ISBN 9781427797995.

[7] Mehta J. B., Dutt A., Harvill L., and K. M. Mathews, "Epidemiology of extrapulmonary tuberculosis: a comparative analysis with pre-AIDS era 1991; 99 (5): 1134-1138.

[8] Akgun E., Tekin F., Ersin S., et al. Isolated perianal tuberculosis. The Netherlands Journal of Medicine 2005; 63 (3): 115-117.

[9] Yurt S., Filiz Kosar A., Isik N et al., "Two cases of pulmonary tuberculosis with extrapulmonary involvement in tongue and anus," Respiratory Medicine Extra 2005; 1 (1): 1-3.

[10] Marshal JB: Tuberculosis of the gastrointestinal tract and peritoneum. Am J Gastroenterol 1993; 88 (7): 989-99.

[11] Todd A. Sheer, Walter J. Coyle: Gastrointestinal tuberculosis. Current Gastroenterology Reports 2003; 5 (4): 273-278.

[12] Donoghue HD, Holton J. Intestinal tuberculosis. Curr Opin Infect Dis 2009; 22: 490.

[13] Mehta J. B., Dutt A., Harvill L., et al. Epidemiology of extrapulmonary tuberculosis: a comparative analysis with preAIDS era. Chest 1991; 99 (5): 1134-1138.

[14] Vanderpool DM, O'Leary PJ. Primary tuberculous enterocolitis. Surg Gynecol Obstet 1988; 167: 167-73.

[15] Horland R. W., Varkey B. Anal tuberculosis: report of two cases and literature review. The American Journal of Gastroenterology 1992; 87: 1488-1491.

[16] Gupta P. J. Ano-perianal tuberculosis-solving a clinical dilemma. African Health Sciences 2005; 5 (4): 345-347.

[17] Gutman H., Kott I., Santo M., et al. Perianal tuberculosis: report of a case and review of the literature. Digestive Surgery 1985; 2 (2): 118-120.

[18] Mathew S. Anal tuberculosis: report of a case and review of literature. International Journal of Surgery 2008; 6 (6): e36e39.
[19] Sultan S., Azria F., Bauer P., et al. Anoperineal tuberculosis: diagnostic and management considerations in seven cases. Diseases of the Colon and Rectum 2002; 45 (3): 407-410.

[20] Petrosyan M, Mason RJ. Tuberculous enteritis presenting as small-bowel obstruction. Clin Gastroenterol Hepatol 2006; 4: xxiii.

[21] Dasgupta A, Singh N, Bhatia A. Abdominal tuberculosis: a histo-pathological study with special reference to intestinal perforation and mesenteric vasculopathy. J Lab Physicians 2009; $1: 56$.

[22] Ha HK, Ko GY, Yu ES, et al. Intestinal tuberculosis with abdominal complications: radiologic and pathologic features. Abdom Imaging 1999; 24: 32.

[23] Ozarmagan G., Keles S., Yazganoglu K. D., et al. Delayed diagnosis in a case of perianal tuberculosis: differential diagnosis in perianal ulceration Indian J Dermatol 2010; 55: 309-310.

[24] Miteva L. and Bardarov E. Perianal tuberculosis: a rare case of skin ulceration? Acta Derm Venereol 2002; 82: 481-482.

[25] Alvarez C. J. L., Gutierre A. V. M. z, Del Riego T. J., et al. Perianal ulcers of tubercular origin. A report of 3 new cases Rev Esp Enferm Dig 1992; 81: 46-48.

[26] Netoa I. J. F. C, Siáa O. N., Lopes E. A., et al. Perianal tuberculosis: A rare disease of late diagnosis. Journal of Coloproctology 2014; 34 (2): 124-127.

[27] Walsh T. J., Mulholland J. H. Laryngeal and perianal tuberculosis simulating laryngeal and perianal carcinoma, Johns Hopkins Medical Journal 1981; 149 (4): 135-137.

[28] Ibn Majdoub Hassani K., Ait Laalim S., Toughrai I. et al. Perianal Tuberculosis: A Case Report and a Review of the Literature. Infectious Diseases 2012; Volume 2012 (2012), Article ID 852763.

[29] Ichihashi K., Katoh N., Takenaka H., et al. Orificial tuberculosis: presenting as a refractory perianal ulcer. Acta Derm Venereol 2004; 84: 331-332.

[30] Sharma M. P., Bhatia V. Abdominal tuberculosis. Indian J Med Res 2004; 120: 305-315.

[31] Shahrokh S, Bagher Miri M., Safari M. T. et al. Pancreatic Tuberculosis: An overview J Pancreas (Online) 2015; 16 (3): 232-238.

[32] Auerbach O. Acute Generalized Miliary Tuberculosis. Am J Pathol 1944; 20: 121-136. [PMID: 19970738].

[33] Nagar AM 1: Pancreatic tuberculosis: a clinical and imaging review of 32 cases). J Comput Assist Tomogr 2009; 33: 136141. [PMID: 19188801].

[34] Khan R, Abid S, Jafri W, et al. Diagnostic dilemma of abdominal tuberculosis in non-HIV patients: an ongoing challenge for physicians. World J Gastroenterol 2006; 12: 6371-5. [PMID: 17072964].

[35] Turan M, Sen M, Koyuncu A, et al. Pancreatic psuedotumor due to peripancreatic tuberculous lymphadenitis. Pancreatology 2002; 2: 561-4. [PMID: 12435870].

[36] Sonthalia N, Ray S, Pal P, et al. Fine needle aspiration diagnosis of isolated pancreatic tuberculosis: A case report. World J Clin Cases 2013; 16 (1): 181-6. [PMID: 24303497]. 
[37] Crowson MC, Perry M, Burden E. Tuberculosis of the pancreas: a rare cause of obstructive jaundice. Br J Surg 1984; 71: 239. [PMID: 6697132].

[38] Teo LL, Venkatesh SK, Ho KY. Clinics in diagnostic imaging. Singapore Med J 2007; 48: 687-692. [PMID: 17609835].

[39] Saluja SS, Ray S, Pal S, et al. Hepatobiliary and pancreatic tuberculosis: a two decade experience. BMC Surg 2007; 7: 10. [PMID: 17588265].

[40] Mert A., Ozaras R., Tabak F., et al. Localized hepatic tuberculosis. Eur J Intern Med 2003; 14: 511-2.

[41] Ferrari T. C. A; Couto C. M.; Vilaça T. S., et al. Localized hepatic tuberculosis presenting as fever of unknown origin: case report. Brazilian Journal of Infectious Diseases 2006; 10 (5): $1-4$.
[42] Tritou I., Prassopoulos P., Daskalogiannaki M., et al. Miliary hepatic tuberculosis not associated with splenic or lung involvement. Acta Radiol 2000; 41: 478-81.

[43] Chien R. N., Lin P. Y., Liaw Y. F. Hepatic tuberculosis: comparison of miliary and local form. Infection 1995; 23: 512.

[44] Hersch C. Tuberculosis of the liver: a study of 200 cases. S Afr Med 1964; 38: 857-63.

[45] Kok K. Y. Y., Yapp S. K. S. Isolated hepatic tuberculosis: report of five cases and review of the literature. J Hepatobiliary Pancreat Surg 1999; 6: 195-8. 